\title{
Towards 1’000’000 resolved points in a distributed optical fibre sensor
}

\author{
Marcelo A. Soto*, Luc Thévenaz \\ EPFL Swiss Federal Institute of Technology, Institute of Electrical Engineering, \\ SCI STI LT, Station 11, CH-1015 Lausanne, Switzerland \\ *E-mail: marcelo.soto@epfl.ch
}

\begin{abstract}
Increasing the number of resolved points, by either improving the spatial resolution or extending the measurement range, is one of the main challenges being currently faced in distributed optical fibre sensing. This paper explores the main parameters affecting the performance of Brillouin distributed fibre sensors and analyses potential configurations and directions to reach one million equivalent resolved points.
\end{abstract}

Keywords: Optical fibre sensor, stimulated Brillouin scattering, distributed fibre sensor

\section{INTRODUCTION}

For more than two decades many innovative solutions have been proposed to improve the performance of Brillouin optical-time domain analysis (BOTDA) sensors [1]. One of the most interesting features of this method is the possibility to perform distributed measurements covering very long distances, i.e. tens of kilometres, with high spatial resolution. This gives unique opportunities to monitor large structures, in which a huge number of resolved points could be required. Using standard BOTDA schemes the number of resolved points has basically remained below $50 \mathrm{k}$, and generally limited to 20k-30k. However, remarkable progresses in increasing the number of resolved points have been reported in the last decade. On the one hand, the spatial resolution, ultimately limited to $1 \mathrm{~m}$ by the acoustic-wave response time, has been improved down to the centimetre scale using methods based on correlation-domain, acoustic pre-activation, differential pulses, among others [2-5]. On the other hand, the sensing range, essentially limited by the fibre attenuation and the onset of nonlinear effects, has been extended beyond $100 \mathrm{~km}$ using distributed Raman amplification and pulse coding methods [6-10]. This way, only using advanced techniques, the number of points has been increased beyond 100k [8-10], enabling even 300k points for short distances [5]. Nowadays there are intense research activities to further increase the number of resolved points; however, important challenges have to be faced to offer significant improvements and to reach, for instance, 1 million resolved points in a single system. To reach this huge number of points, all effects having negligible impact in short range schemes (usually sub-metre resolution) have to be properly addressed at long distances. This needs a substantial upgrade of currently-deployed systems; e.g. methods that offer a spatial resolution of $1 \mathrm{~cm}$ have to be able to operate up to $10 \mathrm{~km}$ distance, while the resolution of systems reaching $100 \mathrm{~km}$ has to be improved down to $10 \mathrm{~cm}$. This paper presents an analysis of the most important parameters affecting the performance of BOTDA sensors and limiting the maximum attainable number of resolved points. As it will be described, a successful system upgrade actually needs a precise optimisation of all parameters affecting the sensor response [11] and the noise of the system.

In conventional BOTDA sensors, a high-power pulsed pump signal interacts with a low-power continuous wave (CW) probe through stimulated Brillouin scattering (SBS) [1]. The measurement procedure requires the acquisition of temporal traces of the SBS gain that experiences the probe at different frequency offsets. One of the main requirements to avoid measurement distortions is that the sensor has to operate in a small gain regime, avoiding the detrimental impact of pump depletion [12]. Under this small gain approximation, the response of a BOTDA sensor $\Delta P_{s}(z)$ can be written as [11]:

$$
\Delta P_{S}(z)=\frac{g_{B}}{A_{\mathrm{eff}}} P_{s i} \exp (-\alpha L) P_{p i} \exp (-\alpha z) \Delta z,
$$

where $g_{B}$ is the Brillouin gain, $A_{\text {eff }}$ is the fibre effective area, $\alpha$ is the fibre attenuation coefficient, $L$ is the fibre length, $\Delta z$ is the spatial resolution, and $P_{p i}$ and $P_{s i}$ are the input pump and probe powers. Note that the measurement contrast, and consequently the signal-to-noise ratio (SNR), not only depend on the local pump power, but also on the total losses experienced by the probe signal, doubling the effects of attenuation [11]. Thus, since $\Delta P_{s}(z)$ decays exponentially with distance, the performance must be evaluated at the farthest fibre end where the lowest sensor response is measured.

23rd International Conference on Optical Fibre Sensors, edited by José Miguel López-Higuera,

Julian Jones, Manuel López-Amo, José Luis Santos, Proc. of SPIE Vol. 9157, 9157C3

(C) 2014 SPIE · CCC code: 0277-786X/14/\$18 · doi: 10.1117/12.2072358 
Note that Eq. (1) is valid even for sub-metre schemes, provided a proper pre-activation of the acoustic wave [11]. This equation points out that the sensor response $\Delta P_{S}(z)$ is reduced whenever the spatial resolution is improved or the sensing range is extended [11]. Actually, the well-known trade-off between spatial resolution, sensing range and sensor response (affecting the SNR of the traces and thus the measurand resolution) is evident from Eq. (1) and is considered to be the fundamental rule limiting the performance of distributed optical fibre sensors. All these parameters are interrelated in a system, and whenever one of them is improved at least one of the other parameters is downgraded.

The accuracy of BOTDA sensors is ultimately given by the SNR [11]; therefore, a system performance optimisation requires maximising the sensor response and minimising each source of noise. While the noise defines the smallest detectable sensor response (traded-off with the acquisition time needed to perform trace averaging), the extinction ratio (ER) of the pump pulses imposes another limitation to the maximum number of resolved points in a sensor.

\section{LIMITATIONS IMPOSED BY THE PULSE EXTINCTION RATIO}

In actual BOTDA schemes, the pump pulse is shaped by non-ideal intensity-modulating devices (usually an electro-optic modulator, EOM) that leak a small amount of power in the OFF state of the pulse. This small CW leakage component produces a continuous Brillouin interaction, which accumulates along the entire sensing fibre, reducing the measurement contrast of the useful z-dependent Brillouin gain. The sensor response to this leakage component can be expressed as:

$$
\Delta P_{s}^{\text {leak }}=\frac{g_{B}}{A_{\mathrm{eff}}} k^{-1} P_{p i} P_{s i} \exp (-\alpha L) L_{\mathrm{eff}},
$$

where $k$ is the pulse extinction ratio and $L_{\text {eff }}$ is the fibre effective length. This CW SBS interaction gives to the acquired traces a CW background component that is proportional to the mean gain of the fibre, providing no relevant z-dependent information to the sensor response. Thus, the measured spectrum turns out to be given by the superposition of two gain peaks [13]: one of them corresponding to the mean BGS integrated over fibre length, and the other one to the useful local gain. Depending on the strength of $\Delta P_{s}^{\text {leak }}$ with respect to the pulse contribution $\Delta P_{s}^{\text {pulse }}(z)$, given by Eq. (1), significant measurement errors might occur along the fibre, in particular at long distances where $\Delta P_{s}^{\text {pulse }}(z)$ is highly reduced by the fibre losses. To avoid measurements errors, $\Delta P_{s}^{\text {pulse }}(z=L)$ has to be increased with respect to $\Delta P_{s}^{\text {leak }}$. A straightforward solution to increase the pulse ER is the use of semiconductor optical amplifiers [5,9], cascaded EOMs, or RF shaping methods [13], enabling more than $60 \mathrm{~dB}$ ER. Furthermore, for very long distances more sophisticated techniques have also been reported; these are essentially: distributed Raman amplification [7,9] and optical pulse coding [6,8]. In the first case, the fibre attenuation is partially compensated by distributed optical amplification, being possible to reach an ideal fibre transparency under specific conditions [7]. In the second method, the use of multiple pulses substantially increases the sensor response given by Eq. (1), while the CW leakage contribution remains similar to the standard single pulse case. Fixing the condition $\Delta P_{s}^{\text {pulse }}(L) \geq \Delta P_{s}^{\text {leak }}$, the requirements for the pulse ER can be estimated [9] for each method:

$$
k \geq \frac{L_{\mathrm{eff}}}{\Delta z \cdot \exp (-\alpha L)} \quad \text { (3a), } \quad k \geq \frac{L}{\Delta z} \text { (3b), } \quad k \geq \frac{L_{\mathrm{eff}}}{N_{p} \cdot \Delta z \cdot \exp (-\alpha L)} \quad \text { (3c), } \quad k \geq \frac{L}{N_{p} \cdot \Delta z} \quad \text { (3d) }
$$

Equation (3a) describes the ER required in a standard BOTDA scheme, Eq. (3b) corresponds to the case of an ideal transparent fibre, representing the best-case scenario of bidirectional Raman amplification [7,9], Eq. (3c) describes the optical pulse coding method [6] using $N_{p}$ number of pulses (generally, $N_{p}$ is equal to half the number of bits), and Eq. (3d) shows the requirements for a system combining Raman assistance and pulse coding [10]. The best attainable spatial resolution $\Delta z_{\text {best }}$ can be estimated from Eqs. (3a)-(3d) for each case, as shown in Fig. 1(a). The solid curves in the figure represent the best resolution that can be achieved with ER $=40 \mathrm{~dB}$, for the standard (blue curve), ideal Ramanassisted (red curve) and 512bit coded (black curve) BOTDA schemes. The figure points out that the use of an EOM with $\mathrm{ER}=40 \mathrm{~dB}$ provides only limited sensing distances (e.g. a range of $25 \mathrm{~km}$ for a $\Delta z_{\text {best }}=5 \mathrm{~m}$ ), while much longer distances could be achieved (maintaining the resolution) by increasing the pulse ER, for instance up to $50 \mathrm{~dB}$ (dashed lines). It is important to mention that the $\Delta z_{\text {best }}$ shown in Fig. 1 for Raman assistance only represents the case of ideal transparency, and therefore some degradation of the spatial resolution is expected for more realistic scenarios.

It is thus evident that the maximum number of resolved points $N_{e q}=L / \Delta z_{\text {best }}$ strongly depends on the pulse ER; thus for a standard EOM of ER $=40 \mathrm{~dB}$, a theoretical maximum of only $N_{e q}=10 \mathrm{k}$ points is attainable in the case of ideal fibre transparency. This number actually turns out to be highly reduced if the fibre attenuation is not entirely compensated. Based on Eq. (3), Fig. 1(b) shows the ER necessary to reach $N_{e q}=1$ million points. While standard and ideal Ramanassisted schemes need pulses with more than $\mathrm{ER}=60 \mathrm{~dB}$, much lower requirements exist for coding based methods. 

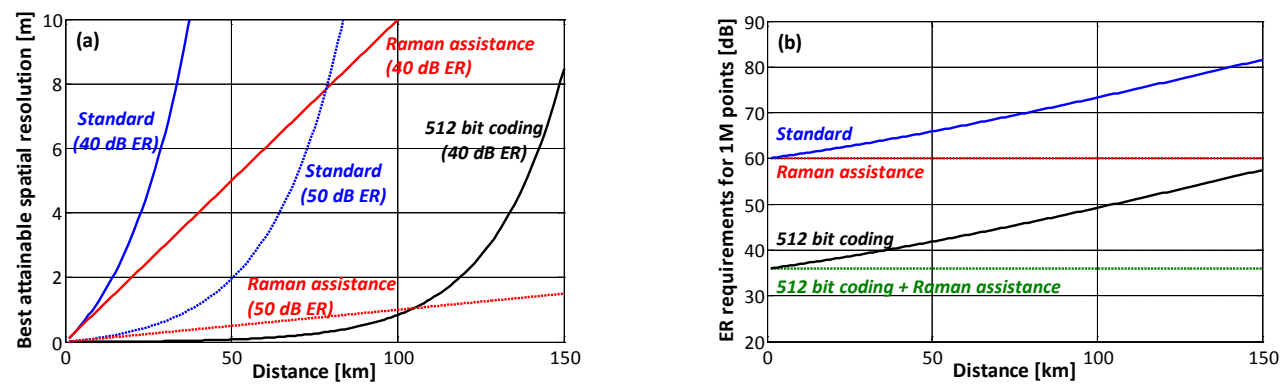

Fig. 1. Effects of pulse ER for different methods: (a) Best attainable spatial resolution. (b) ER requirements for $N_{e q}=1$ million.

Note that these estimations represent limits imposed by the ER when no additional trace is used for correction; thus, differential measurement methods can partially relieve the ER requirements at the expense of longer measurement times.

\section{LIMITATIONS IMPOSED BY NOISE}

Besides the extinction ratio of the pump pulses, the noise level of the system constitutes another fundamental parameter that limits the maximum performance of distributed optical fibre sensors [11]. Considering the presence of an EDFA at the receiver front-end and the influence of relative intensity noise (RIN), the SNR in a BOTDA sensor can be written as:

$$
\operatorname{SNR}(z)=\frac{\Delta I_{s}(z)}{\sigma_{i_{N}}}=\frac{\Delta I_{s}(z)}{\sqrt{\sigma_{i_{t h}}^{2}+\sigma_{i_{S h}}^{2}+\sigma_{i_{S p-s p}}^{2}+\sigma_{i_{s p-s}}^{2}+\sigma_{i_{R I N}}^{2}}},
$$

where $\Delta I_{s}(z)$ is the photocurrent resulting from the sensor response $\Delta P_{s}(z)$ described in Eq. (1), $\sigma_{i_{t h}}^{2}, \sigma_{i_{s h}}^{2}, \sigma_{i_{s p-s p}}^{2}, \sigma_{i_{s p-s}}^{2}$ and $\sigma_{i_{R I N}}^{2}$ are the variance of the photocurrents resulting from thermal noise, shot noise, EDFA spontaneous-spontaneous beat noise, signal-spontaneous beat noise and RIN, respectively. Note that due to the small gain operation [11,12], all kind of power-dependent noises, such as shot noise, signal-spontaneous beat noise and RIN, are essentially given by the probe power reaching the detector without SBS interaction, and are independent of pump power and spatial resolution.

In the vast majority of situations, and assuming optimised probe and pump powers, the thermal noise can be neglected when compared to the shot noise. Only at long ranges the thermal noise could have some impact; however, in such a situation an EDFA is typically used at the receiver to rise the probe power above a minimum detectable level. In absence of any other source of noise, the SNR is expected to be mostly limited by the spontaneous-signal beat noise. However, if the probe is affected by RIN, a significant SNR reduction can be expected, as described in Fig. 2(a), limiting the sensing range considerably. Figure 2(b) shows the maximum distance that secures an accuracy of $1 \mathrm{MHz}$ with a spatial resolution of $1 \mathrm{~m}$, as a function of the averaging number and RIN. It points out that, under usual RIN levels $(>-115 \mathrm{~dB} / \mathrm{Hz})$, it is very difficult to reach more than $40-50 \mathrm{~km}$ in standard schemes. This agrees with the performance reported in the literature, since only using sophisticated methods, the SNR has been improved to allow extended sensing ranges.
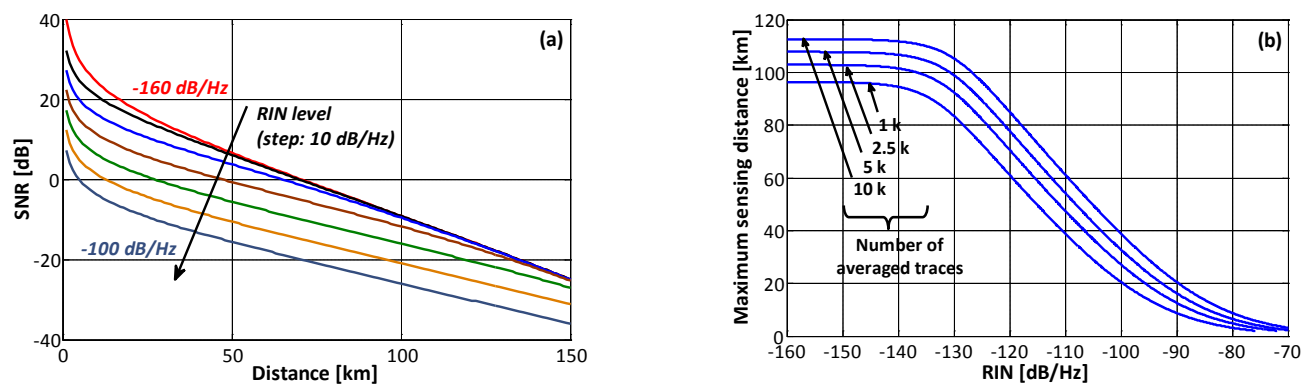

Fig. 2. Effects of RIN on Brillouin sensors with spatial resolution of $1 \mathrm{~m}$ using optimised powers: (a) $\operatorname{SNR}(z=L)$ of single BOTDA trace (at the peak frequency) vs sensing range for different RIN levels. (b) Maximum distance that secures $1 \mathrm{MHz}$ accuracy.

On the other hand, if the spatial resolution in Eq. (1) is adjusted depending on the sensing range, so that a fixed $N_{e q}$ is pre-set, an optimal SNR can be found at medium sensing ranges, as depicted in Fig. 3(a) for the target of $N_{e q}=1$ million resolved points. It worth mentioning that for RIN $>-120 \mathrm{~dB} / \mathrm{Hz}$, this optimal distance tends to $19 \mathrm{~km}$, independently of $N_{e q}$; while lower RIN levels shift the maximum SNR down to shorter distances. Note that in this case the receiver bandwidth can be properly adapted to the spatial resolution, so that the SNR can be suitably optimised. 

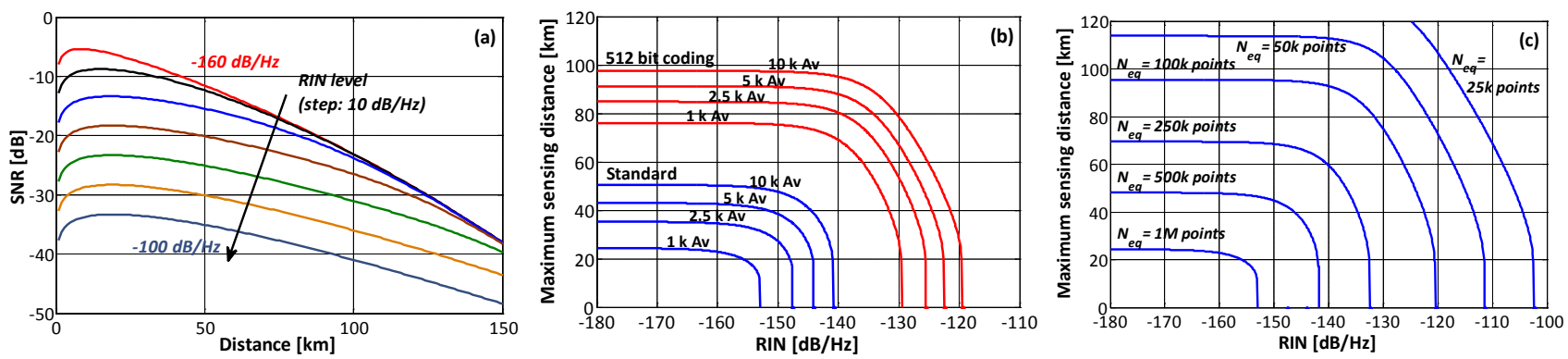

Fig. 3. (a) $\operatorname{SNR}(z=L)$ for $N_{e q}=1$ million vs sensing range for different RIN levels. (b) Maximum range that secures $N_{e q}=1$ million and an accuracy of $1 \mathrm{MHz}$ for different averaging number, in standard (blue curves) and coded (red curves) schemes. (c) Maximum range that secures a given number of resolved points and an accuracy of $1 \mathrm{MHz}$ (with 1k averages) as a function of the RIN level.

Figure 3(b) shows the maximum range that secures $N_{e q}=1$ million and an accuracy of $1 \mathrm{MHz}$ versus RIN and averaging number, with a standard (blue curves) and 512 bit coded (red curves) scheme. It can be seen that $N_{e q}=1$ million points can eventually be obtained only at short-to-medium ranges, requiring RIN below $-140 \mathrm{~dB} / \mathrm{Hz}$, unless special techniques are employed. While for very low RIN levels, the attainable distance is basically constant, as soon as the RIN starts to be the dominating factor, the maximum range is abruptly reduced. This is also observed if a lower $N_{e q}$ is required, as shown in Fig. 3(c). The figure shows that with usual RIN levels ( $>-115 \mathrm{~dB} / \mathrm{Hz}), N_{e q}=100 \mathrm{k}$ can only be reached at short ranges if a large number of averages ( $>1 \mathrm{k})$ is used, being in agreement with existing results. Actually, 100k points have been so far obtained only within a few km [3,4], while for long ranges $N_{e q}$ has remained below 50k, even if advanced methods are used [6]. More than 100k points have been demonstrated at long ranges only by combining Raman amplification and signal processing [8-10].

Finally, Fig. 4 shows the maximum $N_{e q}$ versus RIN for standard (blue curve) and 512 bit coded (red curve) BOTDA schemes, using $1 \mathrm{k}$ averages. The figure points out that an alternative to significantly increase $N_{e q}$ in a RIN dominated system is to reduce the receiver bandwidth (dashed lines), while keeping a very high spatial resolution. This condition is satisfied by correlation-based methods [2,5], giving the possibility to reach $N_{e q}=1$ million, for instance with a RIN $=-110 \mathrm{~dB} / \mathrm{Hz}$ and a detection bandwidth lower than a few MHz.

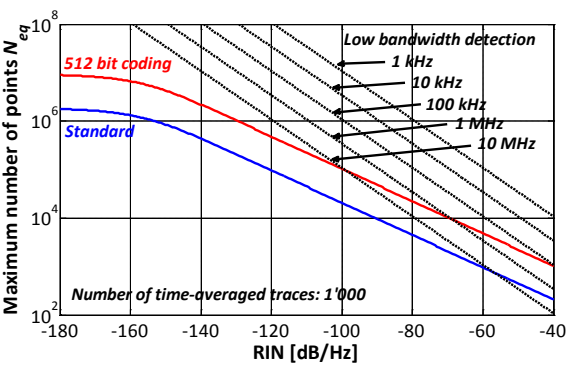

Fig. 4. Maximum number of points vs RIN for standard (red curve), coding based (blue curve) and low-bandwidth detection (black dashed lines) Brillouin sensing schemes.

In conclusion, the challenging objective to reach 1 million resolved points in a single Brillouin sensor undoubtedly requires innovative solutions to get rid of fundamental limitations, imposed essentially by the pulse extinction ratio and relative intensity noise. A smart combination of existing methods for high spatial resolution and long sensing distances could increase significantly the number of resolved points in currently-deployed Brillouin sensors, giving the possibility to reach 1 million points under particular circumstances, for instance using low-frequency detection schemes. Novel alternative methods could eventually lead to huge number of points with less restrictive conditions.

\section{REFERENCES}

[1] T. Horiguchi et al., J. Lightwave Technol. 13(7), 1296-1302 (1995).

[2] K. Hotate et al., IEEE Photon. Technol. Lett. 14(2), 179-181 (2002).

[3] S. M. Foaleng et al., J. Lightwave Technol. 28(20), 2993-3003 (2010).

[4] Y. Dong et al., Applied Opt. 51 (9), 1229-1236 (2012).

[5] A. Denisov et al., Proc. SPIE 8794, Fifth European Workshop on Optical Fibre Sensors, 87943I (2013).

[6] M. A. Soto et al., Opt. Lett. 35(2), 259-261 (2010).

[7] S. Martin-Lopez et al., Opt. Express 18(18), 18769-18778 (2010).

[8] M. A. Soto et al., Opt. Express 20(7), 6860-6869 (2012).

[9] X. Angulo-Vinuesa et al., Opt. Express 20(11), 12147-12154 (2012).

[10] M. A. Soto et al., IEEE Photon. Technol. Lett. 24(20), 1823-1826 (2012).

[11] M. A. Soto et al., Opt. Express 21(25), 31347-31366 (2013).

[12] L. Thévenaz et al., Opt. Express 21(12), 14017-14035 (2013).

[13] A. Zornoza et al., Meas. Sci. Technol. 21(9), 094021 (2010). 